\title{
The Future X-ray Observations of Pulsars and Isolated Neutron Stars
}

\author{
Joachim Trümper \\ Max-Planck-Institut für extraterrestrische Physik
}

\begin{abstract}
During the last decade the X-ray observations ROSAT and ASCA have made it possible to distinguish between photospheric (thermal) and (nonthermal) magnetospheric radiation of single neutron stars. The next generation of X-ray satellites (Chandra, XMM and Astro-E) and the follow-up mission Constellation and XEUS- will allow spectroscopic and timing studies with much higher sensitivity and resolution. Primary future goals are to determine the chemical composition of neutron star photospheres and to measure the gravitational redshift of spectral features.
\end{abstract}

\section{Introduction}

Currently we know about 45 "isolated" $\mathrm{x}$-ray emitting neutron stars which can be divided in three categories:

- Radio pulsars showing pulsed or unpulsed $x$-ray emission. In most cases we see nonthermal radiation characterized by power law spectra, and an X-ray luminosity at $1 \mathrm{keV}$ which is proportional to $\dot{E}: \mathrm{L}_{x}(0.1-2.4 \mathrm{keV}) \sim 10^{-3} E$ (ref. 1). In four cases (Geminga pulsar, PSR 0656+14, PSR 1055-52, and possibly Vela Pulsar) an additional blackbody-like component is seen with spectral temperatures below 1 Million $K$, which is interpreted as the photospheric radiation. The measured temperatures are consistent with expectations from standard cooling models. In many other cases this soft component cannot be detected because of interstellar (cold gas) absorption.

- Point sources near the center of young supernova remnants showing blackbody-like X-ray spectra. Such sources have been found in RCW 103, PKS 1209-63 and Puppis-A. Their emission can be interpreted in terms of photospheric radiation from a cooling neutron star.

- Field sources with very soft X-ray spectra and large flux ratios fx/fopt (RX J1856-37, RX J0720-31 and a few others). These sources may be slowly moving neutron stars accreting from ISM or -more likely-cooling neutron stars old enough ( $\left.\geq 10^{5} \mathrm{yrs}\right)$ to have lost their SNR (ref.2). 


\section{The Future}

In the near future new observational capabilities will be provided by Chandra, $\mathrm{XMM}$ and Astro-E, which taken together, will surpass those of ROSAT and ASCA enormously in terms of sensitivity, angular resolution, spectral coverage and spectral resolution.

The specific strengths of these missions relevant to our subject are as follows:

- Chandra: excellent angular resolution: 0.5 arcsec, ten times better than ROSAT

- XMM: large collecting area $\left(\sim 2500 \mathrm{~cm}^{2}\right) \mathrm{cf}$. fig. 1 , ten times better than ROSAT and three times better than Chandra; wide energy band (0.1-10 $\mathrm{keV)}$

- Astro-E: large collecting area combined with very high spectral resolution $(12 \mathrm{eV})$

Since all the pulsars and isolated neutron stars which still have to be discovered will be pretty faint the large collecting power of XMM, in particular that obtained with the pn CCD-detector will be of crucial importance. In particular, the good low energy performance should allow to detect more photospheric emitters.

The thermal radiation from neutron stars photospheres is of great interest for several reasons:

- spectral features produced by heavy elements like iron may be used to measure the gravitational redshift and thus the ratio of mass to radius.

- "photospheric radii" may be measured for sources with known distances, e.g. based on optical parallaxes. This is a real possibility since isolated neutron stars are supposed to be close and within the reach of parallax measurements.

For both topics a knowledge of the spectra expected from neutron star photospheres is important and a lot of theoretical work has been done on this subject recently (e.g. ref. 3-5). Another important aspect of the photospheric emission is that it is either isotropic or only weakly modulated with the rotational period. Therefore, observing a sufficiently large number of such objects and finding out how many of them show up as radio pulsars will be a very direct method to determine beaming factors.

Undoubtedly, Chandra, XMM and Astro-E will dominate the field during the next 5-10 years. On the long run there are two projects offering even larger capabilities: Constellation (NASA) which may be launched in 2008 and XEUS having thousand times larger collecting area $\left(30 \mathrm{~m}^{2}\right)$ than ROSAT which may follow after 2010. At any rate, we anticipate dramatic progress in this exciting field. 


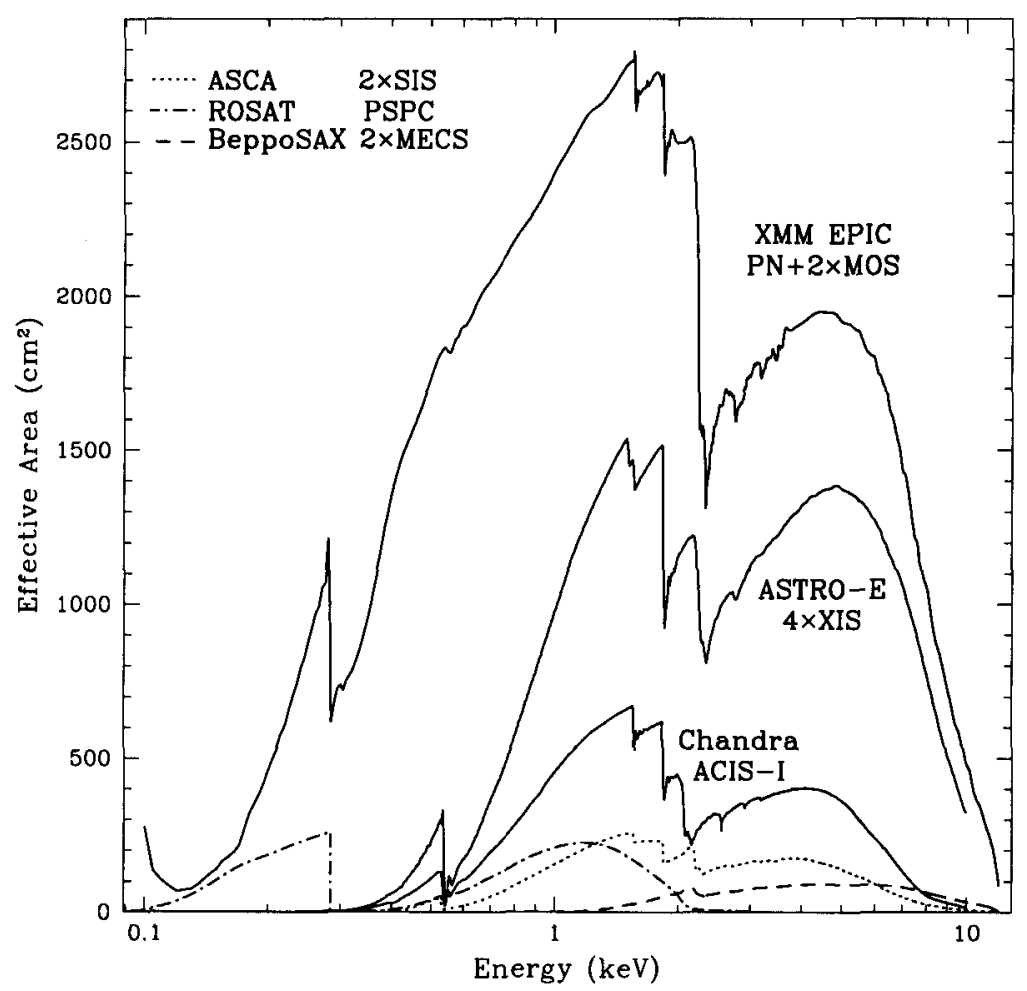

Figure 1. Effective collecting area of recent and current X-ray telescopes satellites 


\section{References}

[1] Becker, W., Trümper, J. 1997, A\&A, 326, 682

[2] Neuhäuser, R., Trümper, J., 1999, A\&A, 343, 151

[3] Romani, R., 1987, ApJ, 313, 718

[4] Zavlin, V.E., Pavlov, G.G., Shibanov, Yu.A., 1996, A\&A, 315, 141

[5] Zavlin, V.E., Pavlov, G.G., Shibaniv, Yu.A., Ventura, J., 1995, A\&A, 297, 441 